\title{
Vaccination against Ostertagia ostertagi with subfractions of the protective ES-thiol fraction
}

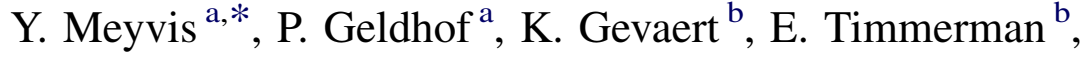 \\ J. Vercruysse ${ }^{\mathrm{a}}$, E. Claerebout ${ }^{\mathrm{a}}$ \\ ${ }^{a}$ Laboratory of Parasitology, Faculty of Veterinary Medicine, Ghent University, \\ Salisburylaan 133, B-9820 Merelbeke, Belgium \\ ${ }^{\mathrm{b}}$ Department of Medical Protein Research, Flanders Institute for Biotechnology, Ghent University, \\ Albert Baertsoenkaai 3, B-9000 Ghent, Belgium
}

Received 4 June 2007; received in revised form 30 July 2007; accepted 3 August 2007

\begin{abstract}
Previous vaccination trials against Ostertagia ostertagi in cattle have demonstrated the protective capacity of a protein fraction termed ES-thiol, which is enriched for activation-associated secreted proteins (ASPs) and cysteine proteases. In this study, ES-thiol was subfractionated through Q-Sepharose anion exchange chromatography to determine whether the ASPs and/or the cysteine proteases are responsible for the induced protection. Calves (seven/group) were immunized three times intramuscularly with $100 \mu \mathrm{g}$ of ES-thiol or equivalent amounts of an ASP-enriched fraction, a cysteine protease-enriched fraction or a rest fraction, with QuilA adjuvant. A negative control group only received QuilA. After the final immunization the animals were challenged with a trickle infection of 25,000 infectious L3 larvae (1000 L3/day; 5 days/week). During a 2-month period the geometric mean cumulative faecal egg count (FEC) of the ES-thiol group was reduced by $62 \%$ compared to the QuilA control group $(P<0.05)$. Groups injected with the ASP-enriched, the cysteine protease-enriched and the rest fraction demonstrated a reduction in cumulative FEC of 74, 80 and 70\%, respectively $(P<0.01)$. Although no significant reductions in worm burdens were observed, adult male and female worms were significantly smaller in all vaccinated groups $(P<0.05)$, except for male worms from the ES-thiol group. These results suggest the protective capacity of ASPs and the presence of other protective antigens in the ES-thiol fraction.
\end{abstract}

(C) 2007 Elsevier B.V. All rights reserved.

Keywords: Ostertagia ostertagi; Activation-associated secreted proteins; Cysteine proteases; Vaccine; Cattle

\section{Introduction}

The gastrointestinal nematode Ostertagia ostertagi is responsible for large losses in cattle productivity. Currently, control is mainly based on the use of anthelmintics. However, the threat of anthelmintic resistance encourages us to look for other means of control, such as vaccination. Previous vaccination trials

\footnotetext{
* Corresponding author. Tel.: +329264 73 87; fax: +329264 7496. E-mail address: yves.meyvis@ugent.be (Y. Meyvis).
}

have shown that immunizing calves with excretionsecretion (ES) material from adult Ostertagia worms affinity purified on a thiol-sepharose column results in a significant reduction of 56-60\% in cumulative faecal egg counts (FECs) during 2 months after a first infection with L3 larvae. This reduction was accompanied by a significant reduction in worm length (Geldhof et al., 2002, 2004). The most abundant proteins in this ESthiol fraction have been identified as activationassociated secreted proteins (ASPs) (Geldhof et al., 2003). ASPs belong to a group of evolutionary related secreted proteins called the SCP/tpx-1/Ag5/PR-1/Sc7 
family and are present in different organisms such as plants, mammals and nematodes. ASP molecules have previously shown their protective capacity in vaccine trials against Ancylostoma caninum (Ghosh et al., 1996; Ghosh and Hotez, 1999; Sen et al., 2000), A. ceylanicum (Goud et al., 2004; Mendez et al., 2005), Onchocerca volvulus (MacDonald et al., 2004) and Haemonchus contortus (Schallig et al., 1997a,b; Vervelde et al., 2002). The exact function of the ASPs remains unclear, although it is believed that they play a role in parasite infection and transition to parasitism (Hawdon et al., 1996). Two ASP molecules of O. ostertagi have been described in ES-thiol, i.e. Oo-ASP-1 and Oo-ASP-2, with a molecular weight around $30 \mathrm{kDa}$ (Geldhof et al., 2003).

Besides the ASPs, ES-thiol contains multiple cysteine proteases with cathepsin L-like activity (Geldhof et al., 2002). The function of these enzymes is unknown, but they might enable the parasite to destroy host tissue for feeding and penetration, and to evade the host immune system (Tort et al., 1999). The protective capacity of cysteine proteases has already been demonstrated against Fasciola hepatica (Wijffels et al., 1994; Dalton et al., 1996) and H. contortus (Boisvenue et al., 1992; Knox et al., 1999, 2005; Redmond and Knox, 2004).

At the moment it is unclear which of these ES-thiol components are essential for rendering protection against an $O$. ostertagi challenge infection, i.e. the ASPs, the cysteine proteases or other so far unidentified proteins present in the ES-thiol fraction. The objective of the present study was to further fractionate the ESthiol fraction in its different components and determine their protective capacity separately.

\section{Materials and methods}

\subsection{Antigen fractionation}

The protective ES-thiol fraction was purified as described previously (Geldhof et al., 2002, 2004). A QSepharose column (Amersham, $1 \mathrm{ml}$ bedvolume) was equilibrated in $10 \mathrm{mM}$ Tris-HCl, $\mathrm{pH}$ 7.4. ES-thiol was applied to the column $(1 \mathrm{ml} / \mathrm{min})$. Unbound proteins were collected and bound proteins were eluted by a stepwise increase in $\mathrm{NaCl}$ concentration (25-50-75100-125-175-250-500-1000 mM). Aliquots of all samples were analysed on a $10 \%$ SDS-PAGE under reducing conditions followed by silver staining. The presence of ASPs was demonstrated by running the samples on a $10 \%$ SDS-PAGE under reducing conditions and blotting onto a PVDF membrane. The blot was blocked for $2 \mathrm{~h}$ in $10 \%$ horse serum in PBS/Tween20 (PBST), probed overnight with rabbit anti-rASP serum obtained by immunization with an E. coli recombinant of Oo-ASP-1 (diluted 1/200 in 5\% horse serum/PBST/ Visser et al., unpublished) and incubated for $2 \mathrm{~h}$ with conjugate (Goat anti-rabbit-HRPO, Sigma; diluted 1/ 5000 in 5\% horse serum/PBST). ASPs were visualized by adding $0.05 \% 3,3$ diaminobenzidine tetrachloride in PBS containing $0.01 \% \mathrm{H}_{2} \mathrm{O}_{2}(\mathrm{v} / \mathrm{v})$. Cysteine proteases were monitored by incubating the samples with synthetic Z-Phe-Arg-AMC as was done in a previously described cathepsin assay (Geldhof et al., 2000). Finally, the material was pooled into three different subfractions, i.e. an ASP-enriched fraction, a cysteine protease-enriched fraction and a rest fraction, followed by a concentration step on an Amicon Ultra-15 membrane (Millipore; 10,000 MWCO). Protein concentrations were determined using the BCA method (Pierce Chemical Co., Rockford, IL, USA). Ten micrograms of ES-thiol and its subfractions were analysed by Coomassie Blue Staining under reducing conditions. The presence of ASP was verified using the same Western blot as described above. Cysteine protease activity was detected by gelatin substrate gel under non-reducing conditions (Geldhof et al., 2000).

\subsection{Vaccination trial}

The vaccination trial was designed as previously described (Geldhof et al., 2002, 2004). A Holstein crossbreed population of 35 female helminth-free calves ( 8 months of age) was randomly divided in five groups of seven animals. All animals were immunized three times intramuscularly in the neck with a 3-week interval. One group received $100 \mu \mathrm{g}$ of ES-thiol per immunization in combination with $750 \mu \mathrm{g}$ of QuilA adjuvant. One group received the same amount of QuilA with Tris-buffer instead of antigen (i.e. negative control group). Three remaining groups were immunized with the ASPenriched, cysteine protease-enriched and rest fraction, respectively. The quantity of each subfraction injected was equivalent to the respective amount received by the group injected with ES-thiol. Serum was taken from each animal before the first immunization and 1 week after the second immunization. The animals were challenged with a trickle infection of 25,000 infectious L3 larvae (1000 L3/day; 5 days/week) which started at the day of the third immunization. All calves were euthanized 3 weeks after the last infection. Parasitological parameters (i.e. FECs, worm counts and worm lengths, percentage of L4 larvae) were analysed as described in previous trials (Geldhof et al., 2002, 2004). 


\subsection{Statistical analysis}

Statistical analysis was performed as described previously (Geldhof et al., 2002, 2004). Data are shown as arithmetic means $( \pm$ S.E.M.) or geometric means (+range). Indicators of worm fitness (i.e. FEC, worm burden and length of adult worms) were expected to be lower in vaccinated animals. A one-tailed MannWhitney $U$-test for pairwise comparison was used to determine significance between group means of vaccinated animals and the adjuvant control. Probability values smaller than 0.05 were considered to indicate significant differences. Furthermore, a Kruskal-Wallis test was performed to demonstrate significance between group means of vaccinated animals. Based on the Bonferroni correction, probability values smaller than 0.01 were considered statistically significant.

\subsection{Antibody response}

The antibody response to the individual antigen preparations was tested using pooled sera from the different groups collected 1 week after the second immunization. Ten micrograms of ES-thiol and its subfractions were separated on a 10\% SDS-PAGE under non-denatured conditions and blotted onto a PVDF membrane. The blot was blocked overnight in $10 \%$ horse serum (diluted in PBST), probed for $2 \mathrm{~h}$ with pooled bovine serum (diluted $1 / 500$ in 5\% horse serum/ PBST), and incubated for $1 \mathrm{~h}$ with conjugate (Rabbit anti-bovine-HRPO, Sigma; diluted $1 / 5000$ in 5\% horse serum/PBST). Recognized proteins were visualized by adding $0.05 \% 3,3^{\prime}$-diaminobenzidine tetrachloride in PBS containing $0.01 \% \mathrm{H}_{2} \mathrm{O}_{2}(\mathrm{v} / \mathrm{v})$.

\section{Results}

\subsection{Antigen fractionation}

The protein composition of ES-thiol and its three subfractions on a coomassie-stained gel is shown in Fig. 1a. The profile of ES-thiol was identical to previously published results (Geldhof et al., 2002, 2004). The ASP-enriched fraction (eluted at 0-25$50 \mathrm{mM}$ ) contained only one visible band at $30 \mathrm{kDa}$. Two protein bands were detected in the cysteine proteaseenriched fraction (eluted at $250-500-1000 \mathrm{mM}$ ) at 28 and $45 \mathrm{kDa}$. The rest fraction (eluted at 100-125$175 \mathrm{mM}$ ) showed multiple protein bands over the entire length of the gel, including a more abundant band at $48 \mathrm{kDa}$.

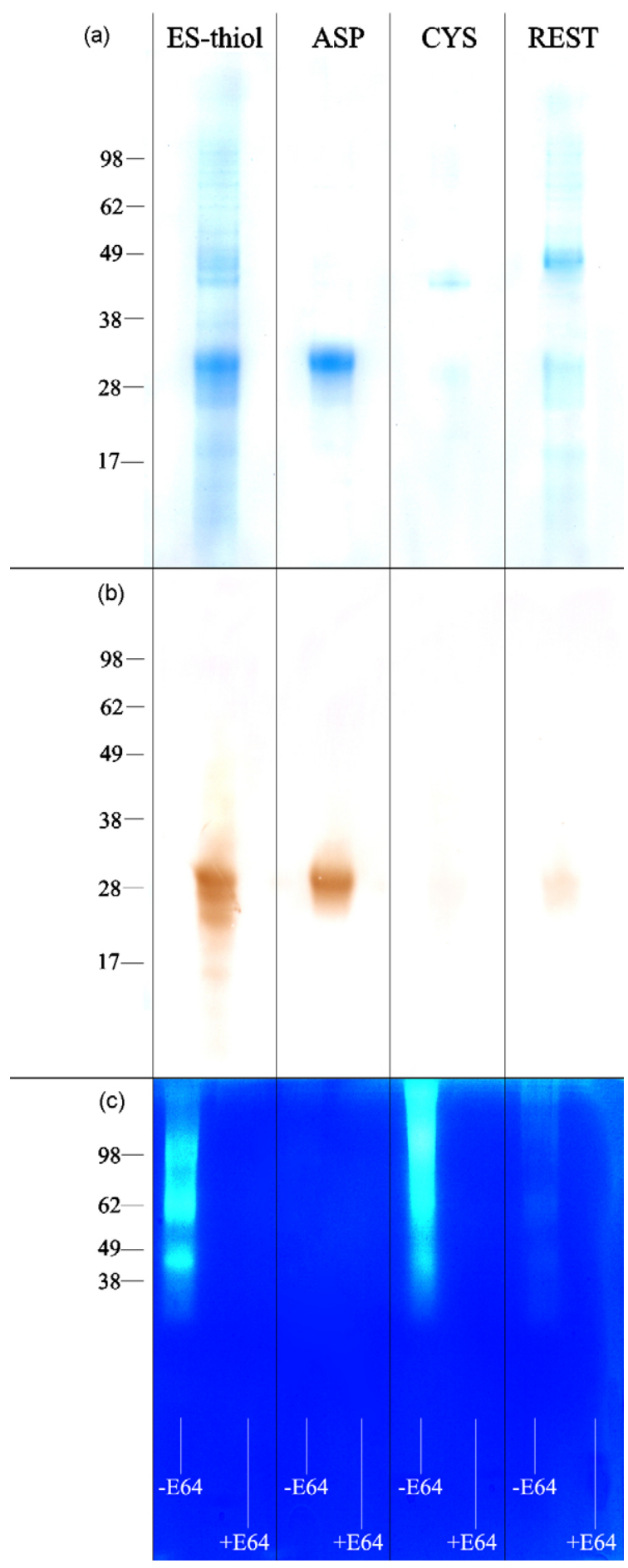

Fig. 1. Analysis of the collected ES-thiol and its subfractionation. (a) Comparison of the protein profiles on a 10\% SDS-PAGE under denaturing conditions, visualized by Coomassie Blue Staining. (b) Detection of ASP proteins using Western blotting and rabbit anti-rASP antibodies. (c) Visualization of cysteine proteases and the effect of the cysteine protease inhibitorE64 on a gelatin substrate gel incubated at $\mathrm{pH} 5.0$ in the presence of $5 \mathrm{mM}$ DTT. Molecular weights of standards are presented in $\mathrm{kDa}$. 
The Western blot analysis with antibodies against ASP is shown in Fig. 1b. The $30 \mathrm{kDa}$ band seen in the ASP-enriched fraction was confirmed to be ASP. No ASP proteins could be detected in the cysteine proteaseenriched fraction. Minor recognition was seen in the rest fraction, possibly indicating trace amounts of ASP.

Fig. 1c shows the cysteine protease activity in the different fractions as detected on a gelatine substrate gel before and after addition of E-64, a cysteine protease inhibitor. Cysteine protease activity present in the ESthiol fraction was highly enriched in the cysteine protease-enriched fraction. The ASP-enriched fraction did not contain any activity, while minor activity was seen in the rest fraction. Addition of an E-64 inhibitor completely abolished protease activity.

\subsection{Vaccination trial}

None of the animals showed adverse reactions to the immunizations and no clinical signs of ostertagiosis were observed. FECs during the vaccination trial are shown in Fig. 2. The geometric mean FEC of all vaccinated groups were lower than the control group throughout the experiment. The parasitological parameters are summarized in Table 1. Immunization with ES-thiol gave a statistically significant reduction in cumulative FEC of $62 \%$. Groups injected with the ASPenriched, cysteine protease-enriched and the rest fraction showed a reduction in cumulative FEC of 74,80 and $70 \%$, respectively. The percentage of L4 larvae was lower than $2 \%$ in all groups and no difference could be seen between the different groups (data not shown). Animals immunized with ASPs and cysteine proteases, respectively contained 47 and $28 \%$ fewer adult worms compared to the controls, although these reductions were not significant. No reduction in worm burden was observed in the ES-thiol group and the rest fraction group. Both female and male worms were significantly smaller in all treated groups with the

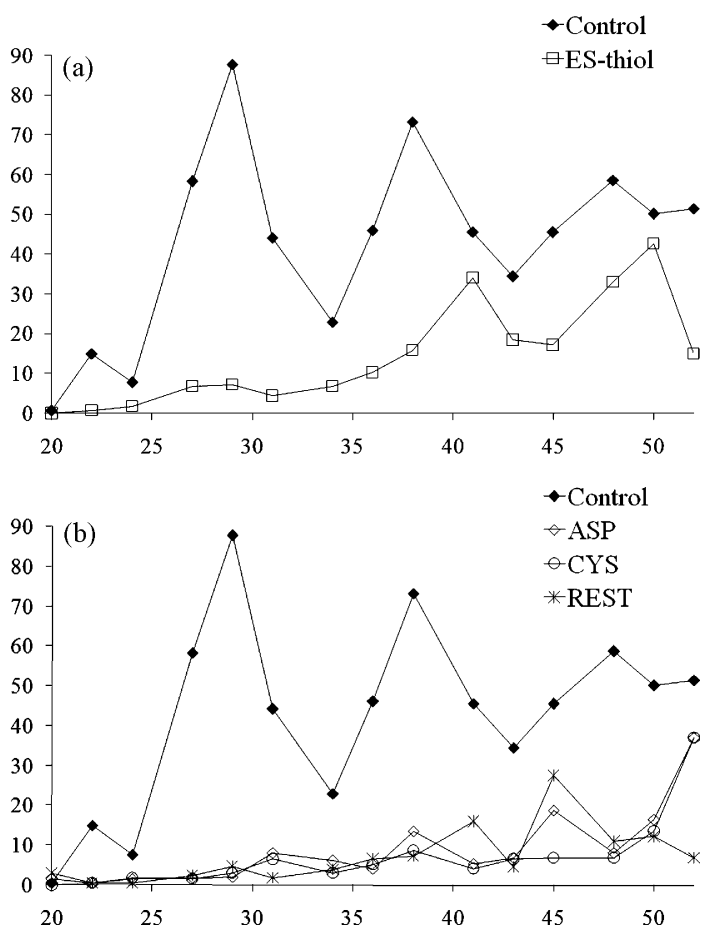

Fig. 2. Geometric mean of the faecal egg output during the 2-month period of the vaccination trial. (a) Animals vaccinated with QuilA versus ES-thiol; (b) groups vaccinated with QuilA versus ASPenriched, cysteine protease-enriched and rest fraction.

exception of male worms found in ES-thiol injected animals. No significant difference was seen between the parasitological parameters of the vaccinated groups.

\subsection{Antibody response}

Fig. 3 shows antibody recognition of non-denatured ES-thiol and its subfractions by pooled sera from each group. Animals injected solely with QuilA did not recognize any ES-thiol proteins.

The ES-thiol group recognized ES-thiol proteins over the entire length of the gel with one immunodominant

Table 1

Parasitological parameters of the vaccination trial

\begin{tabular}{lllllll}
\hline Group & $n$ & Cumulative FEC (EPG) & Reduction $(\%)$ & Number of worms & Worm length $(\mathrm{mm})$ \\
\hline QuilA & 7 & $2034(650-4575)$ & - & $2738(550-8900)$ & F 9.29, M 7.41 & $(8.95-9.92),(6.97-7.92)$ \\
ES-thiol & 7 & $768^{*}(63-2650)$ & 62 & $2891(1450-5050)$ & F 8.26*, M 6.65 & $(6.87-9.11),(5.26-7.71)$ \\
ASP & 7 & $538^{* *}(125-1250)$ & 74 & $1446(400-5400)$ & F 8.22**, M 6.91* & $(7.65-8.91),(6.15-7.57)$ \\
CYS & 7 & $407^{* *}(75-1363)$ & 80 & $1797(100-4500)$ & F 8.14**, M 6.78* & $(7.33-9.03),(5.87-7.70)$ \\
REST & 7 & $618^{* *}(238-2075)$ & 70 & $2536(450-6200)$ & F 8.24**, M 7.03* & $(7.93-8.58),(6.73-7.50)$ \\
\hline
\end{tabular}

F, female, M, male. Number of animals per group ( $n$ ), geometric mean cumulative egg counts (eggs per gram, EPG), total number of worms (geometric mean + range) and worm lengths (geometric mean of 50 worms per animal + range).

${ }^{*} P<0.05$.

*** $P<0.01$. 


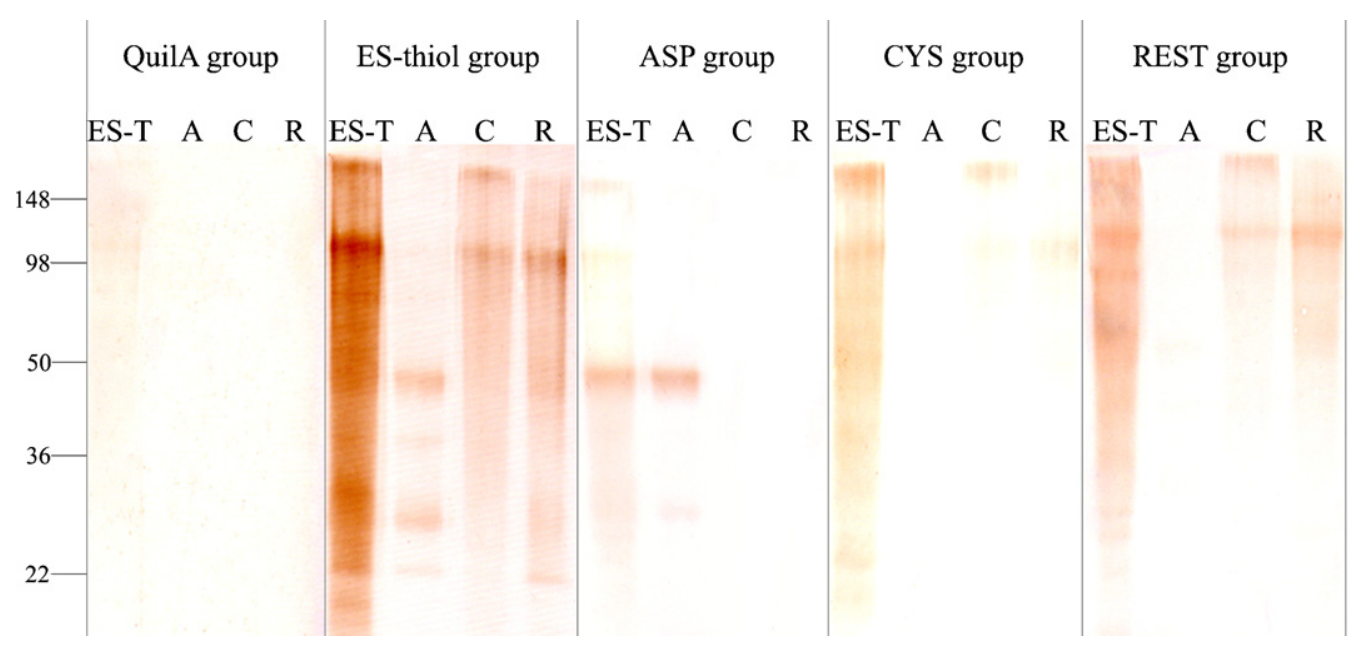

Fig. 3. Detection of serum antibody responses to vaccinations using Western blotting. Sera from individual animals were taken 1 week after the second immunization and pooled for each group (QuilA, ES-thiol, ASP-enriched fraction, cysteine protease-enriched fraction and rest fraction). Lanes were loaded with equivalent amounts of ES-thiol (ES-T), ASP-enriched fraction (A), cysteine protease-enriched fraction (C) and rest fraction (R) in order to detect cross-reactivity.

band at $120 \mathrm{kDa}$. Multiple bands were recognized in the subfractions: two bands at 25 and $50 \mathrm{kDa}$ in the ASPenriched fraction, a band at $120 \mathrm{kDa}$ in the cysteine protease-enriched and the rest fraction, and an additional $250 \mathrm{kDa}$ band in the cysteine protease-enriched fraction.

Animals immunized with the ASP-enriched fraction showed one immunodominant band at $50 \mathrm{kDa}$ and a minor band at $25 \mathrm{kDa}$ in both the ES-thiol and the ASPenriched fraction. These bands were cut from a nondenatured coomassie-stained gel and both were identified as ASP-1 by mass-spectrometry (results not shown). No proteins were recognized in the cysteine protease and rest fraction.

Animals injected with the cysteine protease-enriched fraction and the rest fraction showed a similar pattern. Two high molecular weight bands around 120 and $250 \mathrm{kDa}$ were recognized in the cysteine proteaseenriched fraction. The same $120 \mathrm{kDa}$ band was detected in the rest fraction. Recognition of the $120 \mathrm{kDa}$ band by the rest fraction group was stronger compared to the cysteine protease group. None of the animals recognized proteins from the ASP-enriched fraction.

\section{Discussion}

Published data on successful vaccination trials against O. ostertagi are still scarce. Smith et al. (2000) previously reported a reduction in egg output of $30-50 \%$ by vaccination with gut membrane glycoproteins. More recently, Vercauteren et al. (2004) reported the protective capacity of a secreted polyprotein allergen named OPA. Injection with this ES antigen induced a reduction in faecal egg output of $60 \%$ compared to control animals. Finally, the ES-thiol fraction was previously tested in two independent trials, with the main protective effect being a reduction in cumulative egg counts of 56-60\% (Geldhof et al., 2002, 2004). In the present study, injection with ESthiol resulted in a reduction in cumulative faecal egg counts of $62 \%$, demonstrating the protective capacity of ES-thiol for a third time.

Analysis of the composition of ES-thiol previously indicated the presence of ASPs and cysteine proteases (Geldhof et al., 2002). Both classes of molecules are well known vaccine candidates, which have shown their protective capacity in different host-parasite systems. Animals injected with the ASP-enriched fraction showed a $74 \%$ reduction in cumulative FEC. Analysis of this fraction on gel and Western blot only demonstrated the presence of ASPs. As far as we know, this is the first time native ASPs from any parasite were tested in a vaccine trial in such a pure form. The only other native ASP tested as a vaccine candidate was the $24 \mathrm{kDa}$ single-domain ASP-2 homologue (Hc24) from the sheep parasite $H$. contortus (Schallig et al., 1997b). Adult sheep injected with this protein fraction showed a $77.1 \%$ reduction in mean FEC and a $85.0 \%$ reduction in worm count compared to animals injected with only adjuvant. However, it is unclear if the Hc24 protein itself was actually responsible for the induced protection, since the protein fraction also contained an immunogenic $15 \mathrm{kDa}$ antigen.

The cysteine protease-enriched fraction conferred an $80 \%$ reduction in cumulative $\mathrm{FEC}$, which is the highest level of protection induced against $O$. ostertagi in cattle 
published to date. Three different bands of cysteine protease activity were seen on a gelatine substrate gel, suggesting the presence of at least three different cysteine proteases. Protection induced by purified native cysteine proteases has also been described in vaccination trials against $H$. contortus (Redmond and Knox, 2004; Knox et al., 2005). Sheep injected with cysteine proteases obtained through anion exchange (MonoQ) or affinity chromatography with cystatin (a natural cysteine protease inhibitor) showed a reduction of $28-56 \%$ in mean FEC and a reduction of $33-46 \%$ in worm burden. However, integral membrane cysteine proteases from $O$. ostertagi did not induce protection (Geldhof et al., 2002), although it is not clear whether these cysteine proteases are identical to those present in ES-thiol. Furthermore, it is important to note that the cysteine protease-enriched fraction used in this study also contained other proteins. Therefore, it cannot be concluded that the $O$. ostertagi cysteine proteases alone are responsible for protection. Further purification of this protein fraction is therefore essential. One possibility would be to affinity purify these proteases according to the method described by Redmond and Knox (2004) using the parasite's cystatin as a ligand.

The rest fraction contained the remaining proteins present in ES-thiol and was still a fairly complex mixture. Except for trace amounts of ASPs and cysteine proteases, the other components remain unknown. Protection rendered by this fraction could be attributed to ASPs, cysteine proteases or other so far unknown antigens. Further fractionation combined with a complete proteomics analysis will be essential in order to identify these unknown components and to test their protective capacities separately.

Surprisingly, all subfractions induced a similar level of protection with $70-80 \%$ reduction in cumulative FEC. This might suggest the presence of common protective antigens present in the different subfractions. Although the subfractions were acquired from the anion exchange column under a wide salt gradient, one cannot exclude the possibility that trace amounts of some proteins are present in all fractions. Alternatively, multiple protective antigens could be present in the different subfractions. For this reason, serum cross recognition of the different groups was analysed on Western blot. The animals injected with the ASP-enriched fraction did not show any cross-reactivity with other subfractions and only recognized the ASPs. This observation, combined with the protein profile of the ASP-enriched fraction, strongly suggests that the ASPs are the protective component in this fraction. The analysis also indicated the presence of a common $120 \mathrm{kDa}$ protein in both the cysteine protease- enriched and rest fraction. Unfortunately, this protein could not be visualized by coomassie or silver staining. Therefore, it could not be isolated for mass-spectrometry and its identity still remains unknown.

The results presented in this study strongly suggest that vaccination of calves with native $O$. ostertagi ASPs induces a protective immune response against a homologous challenge infection. This will enable us to compare the protective capacity of future recombinant ASPs to that of its native form. Recombinant ASP molecules have been evaluated for A. caninum (Ghosh et al., 1996; Ghosh and Hotez, 1999; Sen et al., 2000), A. ceylanicum (Goud et al., 2004; Mendez et al., 2005), O. volvulus (MacDonald et al., 2004) and $H$. contortus (Vervelde et al., 2002). These recombinants were produced in bacterial systems and the levels of protection varied between 21 and $79 \%$ reduction in parasite burden. Although bacterial recombinants of parasite antigens have been used previously, expression of cysteine-rich antigens often results in the production of inclusion bodies, making it difficult to solubilise the antigen. Another problem might be the incorrect folding of the ASPs, disabling the formation of possible immunogenic epitopes and preventing proper recognition by the host. Switching to eukaryotic expression systems such as yeast, insect or mammalian cells might solve this problem. For example, Ac-ASP-1 and AcASP-2 were expressed in Pichia pastoris to render soluble recombinant antigens at high yield and low cost (Goud et al., 2004). Besides protein conformation, the glycan structures present on the ASPs may also be crucial for protection. Two putative $N$-glycosylation sites have been described in $O$. ostertagi ASP-1 and ASP-2 (Geldhof et al., 2003). The glycan structures present on these sites might be important for the recognition by the host immune system or essential in the correct folding or the dimerization of the ASPs. In this trial, animals immunized with the native ASPs recognized two different forms: an immunodominant dimeric form at $50 \mathrm{kDa}$ and a monomer at $25 \mathrm{kDa}$. The dimerization of ASP molecules might result in the creation of additional epitopes, explaining its higher immunogenicity on Western blot. Further research on the protein conformation and glycosylation of the ASPs will give valuable information to help in the selection of an appropriate recombinant expression system.

\section{Acknowledgements}

Y.M. is funded by a BOF Grant from Ghent University (no. 01109405) and P.G. is financed by a Marie Curie Reintegration Grant (no. 028860) and is 
recipient of a post-doctoral fellowship of the Fund for Scientific Research Flanders (F.W.O.-Vlaanderen, Belgium). The authors would like to thank Intervet for their financial assistance.

\section{References}

Boisvenue, R.J., Stiff, M.I., Tonkinson, L.V., Cox, G.N., Hageman, R., 1992. Fibrinogen-degrading proteins from Haemonchus contortus used to vaccinate sheep. Am. J. Vet. Res. 53, 1263-1265.

Dalton, J.P., McGonigle, S., Rolph, T.P., Andrews, S.J., 1996. Induction of protective immunity in cattle against infection with Fasciola hepatica by vaccination with cathepsin L proteinases and with hemoglobin. Infect. Immun. 64, 5066-5074.

Geldhof, P., Claerebout, E., Knox, D.P., Agneessens, J., Vercruysse, J., 2000. Proteinases released in vitro by the parasitic stages of the bovine abomasal nematode Ostertagia ostertagi. Parasitology 121, 639-647.

Geldhof, P., Claerebout, E., Knox, D., Vercauteren, I., Looszova, A., Vercruysse, J., 2002. Vaccination of calves against Ostertagia ostertagi with cysteine proteinase enriched protein fractions. Parasite Immunol. 24, 263-270.

Geldhof, P., Vercauteren, I., Gevaert, K., Staes, A., Knox, D.P., Vandekerckhove, J., Vercruysse, J., Claerebout, E., 2003. Activation-associated secreted proteins are the most abundant antigens in a host protective fraction from Ostertagia ostertagi. Mol. Biochem. Parasit. 128, 111-114.

Geldhof, P., Vercauteren, I., Vercruysse, J., Knox, D.P., van den Broeck, W., Claerebout, E., 2004. Validation of the protective Ostertagia ostertagi ES-thiol antigens with different adjuvantia. Parasite Immunol. 26, 37-43.

Ghosh, K., Hawdon, J., Hotez, P., 1996. Vaccination with alumprecipitated recombinant Ancylostoma-secreted protein 1 protects mice against challenge infections with infective hookworm (Ancylostoma caninum) larvae. J. Infect. Dis. 174, 1380-1383.

Ghosh, K., Hotez, P.J., 1999. Antibody-dependent reductions in mouse hookworm burden after vaccination with Ancylostoma caninum secreted protein 1. J. Infect. Dis. 180, 1674-1681.

Goud, G.N., Zhan, B., Ghosh, K., Loukas, A., Hawdon, J., Dobardzic, A., Deumic, V., Liu, S., Dobardzic, R., Zook, B.C., Jin, Q., Liu, Y., Hoffman, L., Chung-Debose, S., Patel, R., Mendez, S., Hotez, P.J., 2004. Cloning, yeast expression, isolation, and vaccine testing of recombinant Ancylostoma-secreted protein (ASP)-1 and ASP-2 from Ancylostoma ceylanicum. J. Infect. Dis. 189, 919-929.

Hawdon, J.M., Jones, B.F., Hoffman, D.R., Hotez, P.J., 1996. Cloning and characterization of Ancylostoma-secreted protein. A novel protein associated with the transition to parasitism by infective hookworm larvae. J. Biol. Chem. 271, 6672-6678.

Knox, D.P., Smith, S.K., Smith, W.D., 1999. Immunization with an affinity purified protein extract from the adult parasite protects lambs against infection with Haemonchus contortus. Parasite Immunol. 21, 201-210.
Knox, D.P., Smith, S.K., Redmond, D.L., Smith, W.D., 2005. Protection induced by vaccinating sheep with a thiol-binding extract of Haemonchus contortus membranes is associated with its protease components. Parasite Immunol. 27, 121-126.

MacDonald, A.J., Tawe, W., Leon, O., Cao, L., Liu, J., Oksov, Y., Abraham, D., Lustigman, S., 2004. Ov-ASP-1, the Onchocerca volvulus homologue of the activation associated secreted protein family is immunostimulatory and can induce protective anti-larval immunity. Parasite Immunol. 26, 53-62.

Mendez, S., Zhan, B., Goud, G., Ghosh, K., Dobardzic, A., Wu, W., Liu, S., Deumic, V., Dobardzic, R., Liu, Y., Bethony, J., Hotez, P.J., 2005. Effect of combining the larval antigens Ancylostoma secreted protein 2 (ASP-2) and metalloprotease 1 (MTP-1) in protecting hamsters against hookworm infection and disease caused by Ancylostoma ceylanicum. Vaccine 23, 3123-3130.

Redmond, D.L., Knox, D.P., 2004. Protection studies in sheep using affinity-purified and recombinant cysteine proteinases of adult Haemonchus contortus. Vaccine 22, 4252-4561.

Schallig, H.D., van Leeuwen, M.A., Cornelissen, A.W., 1997a. Protective immunity induced by vaccination with two Haemonchus contortus excretory secretory proteins in sheep. Parasite Immunol. 19, 447-453.

Schallig, H.D., van Leeuwen, M.A., Verstrepen, B.E., Cornelissen, A.W., 1997b. Molecular characterization and expression of two putative protective excretory secretory proteins of Haemonchus contortus. Mol. Biochem. Parasit. 88, 203-213.

Sen, L., Ghosh, K., Bin, Z., Qiang, S., Thompson, M.G., Hawdon, J.M., Koski, R.A., Shuhua, X., Hotez, P.J., 2000. Hookworm burden reductions in BALB/c mice vaccinated with recombinant Ancylostoma secreted proteins (ASPs) from Ancylostoma duodenale. Ancylostoma caninum and Necator americanus. Vaccine 18, 1096-1102.

Smith, W.D., Smith, S.K., Pettit, D., 2000. Evaluation of immunization with gut membrane glycoproteins of Ostertagia ostertagi against homologous challenge in calves and against Haemonchus contortus in sheep. Parasite Immunol. 22, 239-247.

Tort, J., Brindley, P.J., Knox, D., Wolfe, K.H., Dalton, J.P., 1999. Proteinases and associated genes of parasitic helminths. Adv. Parasit. 43, 161-266.

Vercauteren, I., Geldhof, P., Vercruysse, J., Peelaers, I., Van den Broeck, W., Gevaert, K., Claerebout, E., 2004. Vaccination with an Ostertagia ostertagi polyprotein allergen protects calves against homologous challenge infection. Infect Immun. 72, 2995-3001.

Vervelde, L., Van Leeuwen, M.A., Kruidenier, M., Kooyman, F.N., Huntley, J.F., Van Die, I., Cornelissen, A.W., 2002. Protection studies with recombinant excretory/secretory proteins of Haemonchus contortus. Parasite Immunol. 24, 189-201.

Wijffels, G.L., Salvatore, L., Dosen, M., Waddington, J., Wilson, L., Thompson, C., Campbell, N., Sexton, J., Wicker, J., Bowen, F., Friedel, T., Spithill, T.W., 1994. Vaccination of sheep with purified cysteine proteinases of Fasciola hepatica decreases worm fecundity. Exp. Parasitol. 78, 132-148. 